\title{
Research on Workshop-Based Positioning Technology Based on Internet of Things in Big Data Background
}

\author{
FengChun Liu, ${ }^{1}$ YaLou Liu $\left(\mathbb{D},{ }^{2}\right.$ DongHao Jin $\mathbb{D}^{3},{ }^{3}$ XueYong Jia, ${ }^{3}$ and TingTing Wang ${ }^{3}$ \\ ${ }^{1}$ College of Qianan College, North China University of Science and Technology, Tangshan 063000, China \\ ${ }^{2}$ College of Communication Operations, North China University of Science and Technology, Tangshan 063000, China \\ ${ }^{3}$ The Key Laboratory of Engineering Calculation in Tangshan City, North China University of Science and Technology, \\ Tangshan 063000, China
}

Correspondence should be addressed to YaLou Liu; liuyalou@ncst.edu.cn

Received 13 June 2018; Revised 17 August 2018; Accepted 27 September 2018; Published 15 October 2018

Guest Editor: Zhihan Lv

Copyright (C) 2018 FengChun Liu et al. This is an open access article distributed under the Creative Commons Attribution License, which permits unrestricted use, distribution, and reproduction in any medium, provided the original work is properly cited.

\begin{abstract}
This paper first analyzes the data collection and data management of the workshop, obtains the data of the workshop changes with time, and accumulates the data. There are bottleneck problems such as big data being difficult to be fully used. Then, the concept of the Internet of Things was introduced into the workshop positioning to realize the comprehensive use of the big data in the workshop. Finally, aiming at the positioning problem of manufacturing workshop items, the ZigBee positioning algorithm, the received signal strength indication algorithm RSSI and the trilateration algorithm, is applied, and the trilateral positioning algorithm is applied to the CC2430 wireless MCU, and the positioning node is designed and implemented. The three-sided localization algorithm was used to locate and simulate the horizontal and vertical comparisons of six groups of workshop terminals. The results showed that the difference between the simulated position and the actual position did not exceed $1 \mathrm{~m}$, which was in line with the positioning requirements of the workshop.
\end{abstract}

\section{Introduction}

As we all know, China is a manufacturing country with the name of "World Factory." However, the big data in the workshop and the difficulty of data transmission have caused the weak link between the management of the manufacturing enterprise and the production control of the bottom layer. The upper management can plan and the lower workshop can produce, and this lacks a good convergence, which seriously restricts the competitiveness of the enterprise. Manufacturing execution system (MES) [1] is an important link between the enterprise management planning layer and the field control layer. It effectively achieves the seamless connection between the management plan layer and the field control layer. It can make up for the management defect of the traditional enterprise management mode for big data and make the enterprise more capable, organize production effectively, and improve the company's ability of stochastic adaptability. The implementation of MES must establish a network covering the entire production site. Tracking and positioning must also be implemented using a wireless network. Location data and production data must be integrated and uploaded using a wired network. Scholars such as Chai Jun and Fan Chongyuan [2] put forward an intelligent space based on industrial Internet. By using the collected data, the workshop production and logistics are simplified and optimized. Liu Xiangju, Li Jingzhao, and other scholars [3] proposed a textile shop environment intelligent measurement and control system based on the Internet of Things (LoT) and used the improved Newton interpolation algorithm as a fitting function to process the detection data, improving the detection and control the accuracy of the system. Fang Zhiliang et al. [4] integrated personal digital assistants (PDAs) into manufacturing workshops and proposed the Workshop Internet of Things (WIoT) to improve the production information flow capability.

In a word, the introduction of Internet of Things into manufacturing can effectively solve the problem of big 
data and breakthrough the difficulty of locating bottlenecks. However, in the shop location, the technology of Sawtooth Bee locating is very rare. Aiming at the problem of plane location, this paper puts forward the idea of setting up workshop, builds the workshop network through the Internet of things, realizes and tracks the MES communication function, and then uses the locating algorithm [5], receiving signal intensity indication algorithm RSSI and three-edge positioning. The algorithm can obtain high positioning accuracy by CC 2430 , the wireless SCM, and it is very good to use the position of the article in the workshop.

In the following article, the second section mainly describes the workshop positioning big data collection and processing of specific considerations. The third section introduces the concept of the Internet of Things and combines the Internet of Things with the big data. The fourth section is based on the three-edge positioning algorithm to achieve big data network shop location. The fifth section summarizes fully.

\section{Internet of Things-Based MES Workshop Management Big Data}

2.1. Status of Workshop Data Management. In the current workshop production, manufacturing enterprise production planning is usually prepared by someone and in the form of documents to reach the production workshop. The workshop staff start to arrange raw materials and production process and start with the list, while still needing to assist the progress data semifinished products, and finish the number of finished products to input ERP (Enterprise Resource Planning) system [6]. In this case, the planners do not know the work of the site workshop, the workshop has to plan production, once the plan is higher than the production capacity, it will produce the workshop production progress which cannot keep up with the plan, and it is likely to affect delivery time and delivery quality, resulting in direct economic losses and even affecting the future development of enterprises.

In the collection of on-site processing data, the ERP system is manually entered by the workshop staff, with low efficiency, being unreliable, and even the most recently entered data is of the day before or two days before, having lag, and poor in real time. The big data acquisition module of workshop is composed of several intelligent data collectors; the intelligent data collector takes the high performance digital signal controller as the control core, including the pulse signal acquisition module, the real-time clock module, CAN bus transceiver module, the dot matrix display module, the data storage module, and the fault alarm module. Each module will produce big data; for example, the dot-matrix display module will display a large number of machines in the workshop and real-time production of information, so the entire workshop from the beginning to the final completion of the work will produce big data. Considering the fact that these workshop data are scattered in the state of localization management in China, the data management and utilization system of the system is not formed, so it is very difficult to classify and store the large amount of data and to facilitate the query and real-time updating. A lot of data recording and processing work still need to be handled manually every day, and workshop managers or technicians rely only on paperbased materials or personalized network communication to transfer workshop work information; it is difficult to timely understand and manage the workshop in the current work status or real-time tracking of the details of the workshop, which undoubtedly caused the waste of data resources. The lack of data management and the waste of data resources restrict the development of workshop production in our country to a great extent. ERP and MES operating mode and advantages and disadvantages are shown in Figure 1.

The workshop data acquisition module consists of several intelligent data collectors. The intelligent data collector uses a high-performance digital signal controller as the control core, including a pulse signal acquisition module, a real-time clock module, a CAN bus transceiver module, a dot matrix display module, and a data storage module. Each module will generate big data, so the entire workshop will produce big data from the beginning of work to the final completion. Considering that these shop floor data are currently in a state of fragmented local management and do not form a systematic data management and utilization system, it is very difficult to classify and store big data, facilitate query, and update in real time.

\subsection{MES Workshop Data Collection. Workshop produc-} tion data collection objects include materials, semifinished products, equipment, and other "objects"; the purpose of collecting production information is real-time collection of production-related status. MES (manufacturing execution system) [7] specializes in the process of manufacturing information management; its planning and scheduling functions compared to ERP have a qualitative leap.

MES is based on the most real-time feedback information; its scheduling function of the response is faster and more professional, filling the planning layer and the bottom of the control layer gap. With the development of automation technology, now almost all kinds of automation equipment have their own monitoring module (such as PLC), for visualization operation and fault alarm, but a variety of equipment is difficult to achieve a unified network. MES data acquisition equipment function objective: collect the raw data in the production in a certain way and then upload the result through the network and display it at the same time after the initial operation processing. Such devices require a certain amount of processing power, the ability to network and the common communication interface, and so on. Therefore, it is more appropriate to implement the embedded technology in data acquisition equipment.

Automatic data acquisition including material tracking, progress positioning, cumulative records, and other statistics, to understand the situation of the site is the most critical to obtain on-site data, and the data in real-time and detailed, for more favorable analysis and processing, reliable intelligent data collection, and scientific integration is the key to the implementation of MES. The tracking and locating of various material resources in production site is one aspect of collecting data. In other words, the MES data collection should include two kinds of position data and production data, 


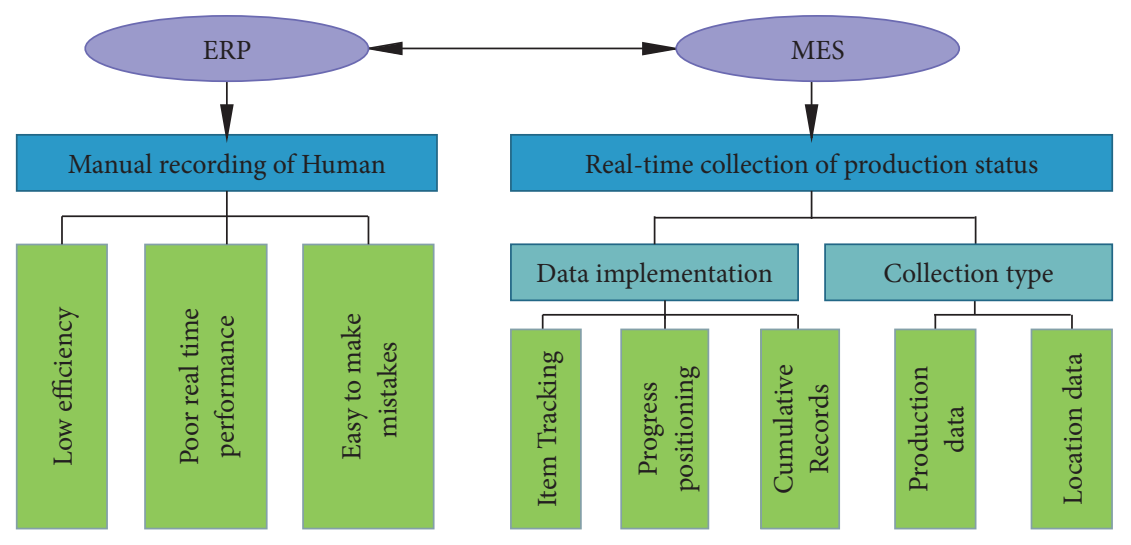

FIGURE 1: Comparison diagram between ERP system and MES system.

and the data acquisition is realized by the specific intelligent data collector. According to the content of the production data acquisition above, the tracking location is undoubtedly realized by wireless network, and the location data and production data need to be uploaded by wired network. Therefore, the demand of the programmable MES network communication must be the entire workshop information network covering the whole production workshop for the integrated wireless network and the wired network.

\subsection{Workshop Internet of Things Data Acquisition and Imple-} mentation. The birth and development of the Internet of Things, to break the one-way contact mode of workshop work, rely on the paper material or personalized network communication to transfer the work of workshop information is gone, so that each module can have timely and comprehensive understanding of the workshop related work progress and general situation, reducing a lot of unnecessary quality problems. The data collected under the network of workshop objects make the unified network between various equipment types, and the advantages of data continuity and consistency are greatly realized.

The realization process of the Internet of Things can be divided into four stages, and the functions of each stage are as follows: The main technologies of object identity identification, information perception, information transmission, and data processing corresponding to each stage function are radio frequency identification technology, sensing technology, network and communication technology, and data mining and fusion technology. The main function of networking is definitely network transmission, but there is also a special data acquisition function in this paper, which is to collect the location information of the object, by laying a special ZigBee wireless network in the workshop and special node with positioning engine, just like the mobile phone with GPS function in life. Not only can the network communication be completed, but also it can be used to determine the location of its own mobile phone when needed for navigation. However, the location in the workshop is very different; location information in the workshop mainly indicates which workshop or which process the material is currently in or whether it has entered the designated warehouse.

Therefore, we implement the positioning of the program mainly in the workshop, the scope of the process, warehouse, and other units for the regional positioning, that is, the workshop on the Internet positioning as long as the accuracy to the region can meet the requirements. The current peer-topeer technology and cloud computing (Cloud Computing) distributed computing technologies have injected new life into the field of data processing and supported the business of IoT. Cloud computing can achieve massive data operation support and can provide remote computing services over the network. The combination of relatively safe and large capacity of data centers and powerful data processing capabilities, at the same time, relying on the Internet strong network transmission capabilities, data security sharing to the terminal, can be effectively implemented. IoT terminal (Internet of Things terminal, iott) is a collection of devices that act as a perceptual layer in the Internet of Things; its task is to collect data and send data to the network layer; its performance is the key index of IoT, the main function is to collect data and transmit it, and it is also necessary to have a simple processing of data for security purposes.

The source function of information system is data acquisition, the real-time reliability of data information is one of the most important characteristics of information system, and the MES system based on information network more strictly collects data as the product of information. The information transmission mode of MES based on IoT changes from mutual gain information to the unified extraction information from the large data platform, and the information acquisition mode changes as shown in Figure 2.

2.4. Internet of Things Data Feature Analysis. The workshop production data is changed with time; the general enterprise will set up workshop data manager to record and classify the workshop data. In fact, the change in workshop data is also traceable, although all data changes over time, but most of the data are cumulative over time; the processing of data can follow the main product specifications, equipment main line, the main process category, and so on. The main line of 


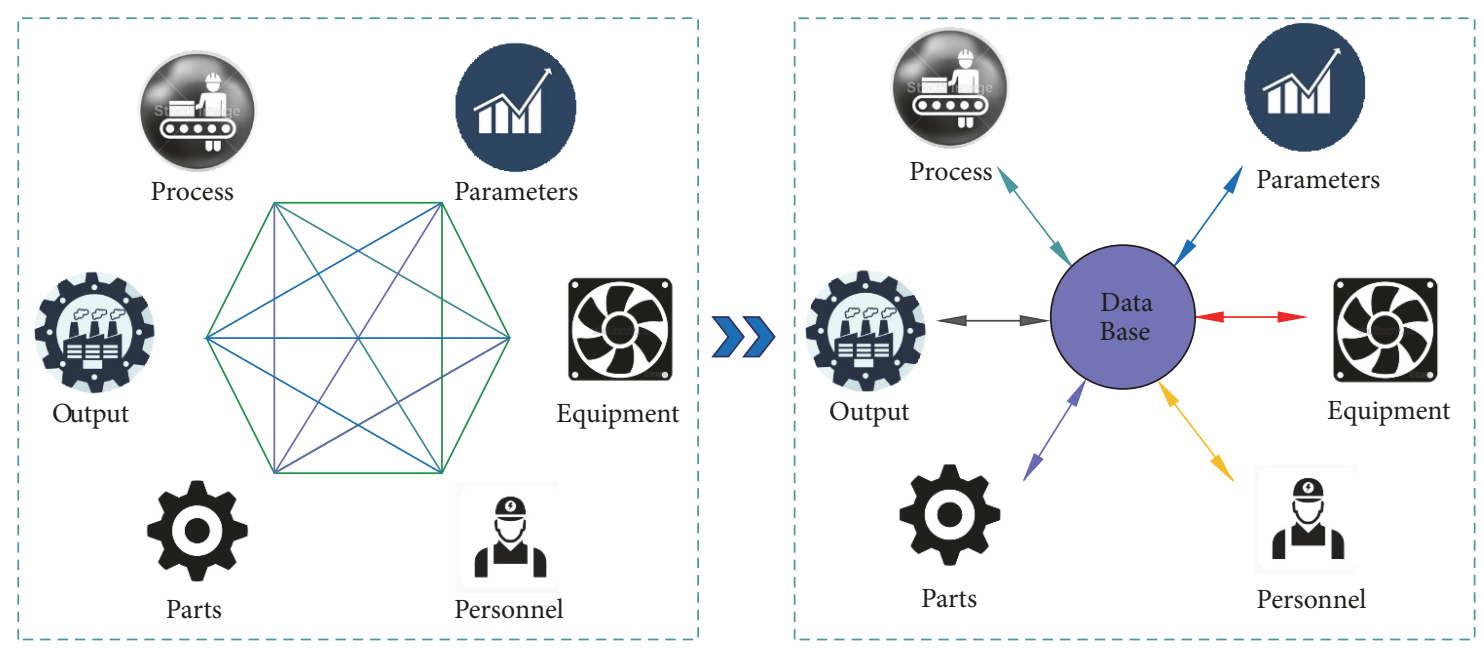

Figure 2: Changes in Information Exchange Models.

product specification is to classify the data according to the specification, and all the data of the same specification will be accumulated with time. The main line of equipment is to monitor the specific equipment; you can get the start-up time of equipment, running state, and cumulative output, at any time to master the production capacity of equipment. The main line of the operation is to record the production data in the way of the cumulative output of each process, and the production data of the same process are summarized together so that the production progress can be understood first.

According to the requirement of actual development and application, the main line can be used as the basis for the identification of the object network node. From the analysis of workshop data characteristics, it can be concluded that the node of the network of workshop materials should be properly arranged and identified by demand. Data processing can be divided into the acquisition end processing and host computer database processing. The processing of the acquisition end is mainly implemented by the embedded technology, so the ability is limited, just some simple filtering, accumulative, percent calculation, and so on. And the processing of the host computer is more complex, mainly to the volume of data statistical analysis, the calculation of production reach rate, production status graphics, and according to the provisions of the report. Of course, all the data processing needs to have the corresponding software algorithm support to complete, so, for different applications, software processing methods are different.

\section{Workshop Internet of Things}

3.1. Meaning of Internet of Things. The Internet of Things [8] is a new product of IT development. The "net" word in the Internet of Things represents the Internet; that is, the Internet is the prototype and conceptual foundation of the Internet of Things. In this article, the network terminal of the thing represents the realization of the shop location. As shown in Figure 3, the ultimate goal of the workshop was that any

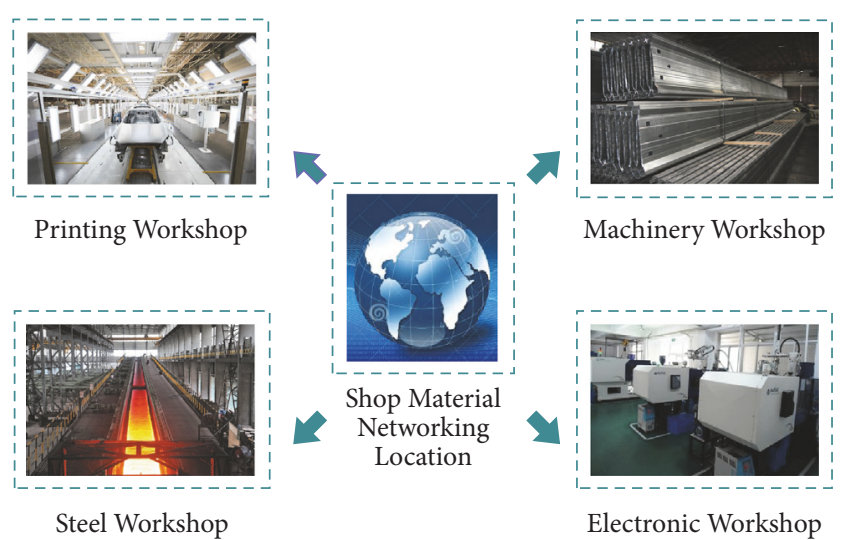

FIGURE 3: Positioning of Workshop Internet of Things.

project could legitimately be positioned at any time, at any place, and on all objects and that all objects could exchange information over the network so that the projects could be connected and constituted the Internet, something in the workshop.

3.2. Workshop Information Network Technology Architecture. In order to implement the positioning technology for the Internet of Things [9] in the workshop, this paper takes the architecture of the Internet of Things as a subdivision and divides it into architecture with very distinct hierarchical features. It is eventually divided into three levels: the perception layer, the network layer, and the application layer, as shown in Figure 4.

(1) Perception layer [10]: as the name implies, it is the first level of object positioning in a workshop, which mainly refers to the collection of position information of the workshop objects. As the basic level of the Internet of Things workshop positioning architecture, the perception layer is the key foundation for the positioning of the Internet of Things. Its function is to realize the automatic identification of physical 


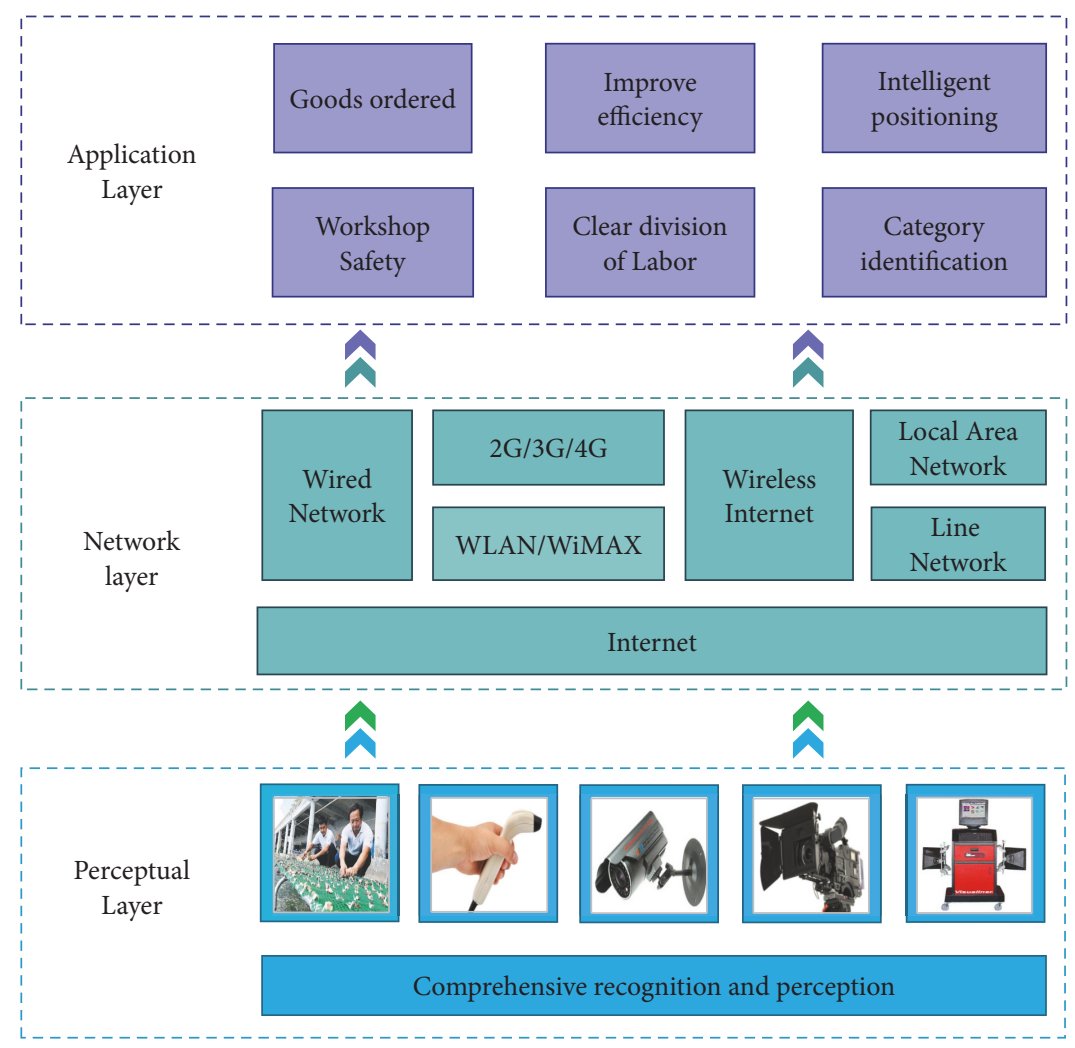

FIGURE 4: Internet of Things technology architecture.

identities, the collection of attributes, and other information exchanges with other levels of information. Sensing facilities in the workshop usually have marking marks, electronic scanning codes, camera sensing, photo recording, and scanning positioning. Based on the perceived characteristics of the object, automatic sensing devices such as radio frequency identification (RFID) and sensors are used to automatically collect accurate position information.

(2) Network layer: the network layer is the core of the positioning of the Internet of Things in the workshop and is the level of mutual transmission of the information of the Internet of Things. It can be understood as an Internet of Things information exchange platform built by the existing wireless network technology and wired network technology. The remaining information can be added to the network through various access devices [11].

(3) Application layer: the practical aspect of the application layer is the realization of the functions after the positioning of the Internet of Things in the workshop, such as workshop security, clear division of labor, classification and identification, ordered items, regular inspections, improved efficiency, and intelligent positioning. This is the ultimate form of presentation of location information data, providing a rich Internet of Things application for platforms that have known locations [12].

In summary, the workshop's Internet of Things is divided into three levels: the perception layer, the network layer, and the application layer. The perception layer helps to obtain the position information of the object, mainly through the number, sensor, etc. to achieve the purpose of information collection. The network layer serves as the middle layer of the IoT architecture and carries the network transmission of comprehensive positioning data collected by the sensing layer. The application layer is the ultimate goal of the Internet of Things and combines the application of the Internet of Things with geographic information.

\subsection{Analysis of IOT Workshop Positioning Terminal Model.} The IoT workshop positioning has various challenges due to the different workshop environment, its perception, and the realization of network transmission. Because of the special location, it is in the convergence of the sensory layer and the network layer. It has an important role in the implementation of the Internet of Things (IoT) device. Only by achieving effective work can all kinds of external information be correctly perceived and organized and sent to designated locations. From different positions of positioning information, positioning is mainly divided into the following three situations: fixed terminals, mobile terminals, and handheld terminals.

Fixed Terminal. The location is fixed for a long time, which can facilitate the acquisition of positioning information. Therefore, video recording can be used to obtain accurate location information, and data transmission can be performed using a wired network, which is reliable and costeffective. 
TABLE 1: Feature comparison items for wireless technology.

\begin{tabular}{|c|c|c|c|c|c|}
\hline & ZigBee & $3 G$ & GPS & RFID & Bluetooth \\
\hline $\begin{array}{l}\text { Application } \\
\text { Scenarios }\end{array}$ & Detection control & $\begin{array}{c}\text { Wide area Range data } \\
\text { transfer }\end{array}$ & Outdoor positioning & $\begin{array}{c}\text { Logistics } \\
\text { Management }\end{array}$ & $\begin{array}{l}\text { Small Range data } \\
\text { transfer }\end{array}$ \\
\hline Cost & Minimum & Higher & Highest & Low & Lower \\
\hline Security & AES 128 High & $\begin{array}{l}\text { High coding } \\
\text { technology }\end{array}$ & PRN Code Higher & Poor CRC code & $\begin{array}{c}\text { Stream Password E0 } \\
\text { high }\end{array}$ \\
\hline Number of nodes & $255 / 65535$ & Many & Many & 2 & 7 \\
\hline Power & Low & Higher & High & - & Higher \\
\hline $\begin{array}{l}\text { Communication } \\
\text { distance }\end{array}$ & $10 \sim 80 \mathrm{~m}$ & $\geqq 1 \mathrm{lkm}$ & $2 \times 104 \mathrm{~km}$ & $1 \sim 10 \mathrm{~m}$ & $1 \sim 10 \mathrm{~m}$ \\
\hline Data transfer Rate & 20/250kbps & $64 \sim 128 \mathrm{kbps}$ & $50 \mathrm{bps}$ & $200 \mathrm{kbps}$ & $1 \sim 3 \mathrm{Mbps}$ \\
\hline
\end{tabular}

Mobile Terminal. Position changes occur at any time and no specific information can be collected. Communication with the outside world can only use wireless network technology. The most vivid examples are mobile phones and computers. Its main functions include awareness of environmental information, location, and wireless communication. This kind of positioning information has low energy consumption, but at the same time it must have good information transmission functions, and due to constant movement, it should also have antiseismic, antifall, and other properties.

Handheld Terminal. Similar to a mobile object, it can be made available. The biggest feature is its small size and portability. It can also be powered by a back-up battery and can operate continuously for more than one day without an external power supply. Information can be exchanged with the outside world through wireless or wired download. It has the ability to sense or read object properties. Such terminal equipment is generally used in fields such as logistics RFID identification [13], factory parameter table inspection, and crop pest and disease survey.

The types of terminals involved in IoT positioning in this article are also in the categories described above. The intelligent data acquisition equipment belongs to the category of industrial equipment testing terminals and fixed equipment terminals and is used to collect the coordinates and position parameters of objects. The wireless network node device based on ZigBee technology belongs to the mobile terminal, and its function is to track and locate the position of the mobile object.

3.4. IOT ZigBee Workshop Positioning Data Analysis. Using the Internet of Things to create a network structure of the objects in the workshop, to monitor every item in real time, because the amount of data in the workshop is too large, we must rely on the Internet of Things. In the specific implementation of positioning problems, based on the network of things to establish a shop location we must focus on the surrounding environment. Due to the large amount of data, various devices were collected on the spot, with strong electricity and weak electrical presence. Therefore, the network of things, especially the wired network, must have the ability to work properly in this environment. The wired network is used to collect location information and object state, and the object to the nearest distance. This paper uses CAN network technology to realize the content of some Internet in wired network. The network part is applied to the realization of wireless network technology. It has powerful network capabilities, is of low cost, and has dedicated hardware and support for wireless network locations. Wireless network plays a role in positionable MES system.

\section{ZigBee Workshop Positioning Based on Internet of Things}

4.1. Comparison of ZigBee Technology and Other Wireless Technologies. The realization of wireless positioning technology in the workshop Internet of Things is the most critical feature of the MES system. This article uses the ZigBee wireless network to implement the network function of location tracking in the Internet of Things. ZigBee is a low-power personal area network protocol based on the IEEE802.15.4 standard [14]. This protocol is a very practical wireless communication network protocol. The SoC equipped with ZigBee protocol is small in size, simple in circuit, and easy to embed in various development applications. Because of its low power consumption and convenient networking, it is widely used in industrial control and remote monitoring and other fields. In short, ZigBee can be seen as a simple and practical economical wireless communication technology. Table 1 gives a comparison of the features of various wireless technologies and briefly describes the wireless technology differences [15] used by various location technologies in the Internet of Things.

From the analysis of Table 1, you can get the following: ZigBee technology is more suitable for the positioning of scenic spots in a specific outdoor area, with the advantages of strong self-organization and high scalability. ZigBee compares the advantages of positioning algorithms for indoor positioning problems:

(1) In the civilian field, the positioning accuracy of GPS is about 10 meters, and the positioning accuracy is relatively low.

(2) The 3G-based positioning technology mainly uses the narrower LBS location service implementation [16]. 

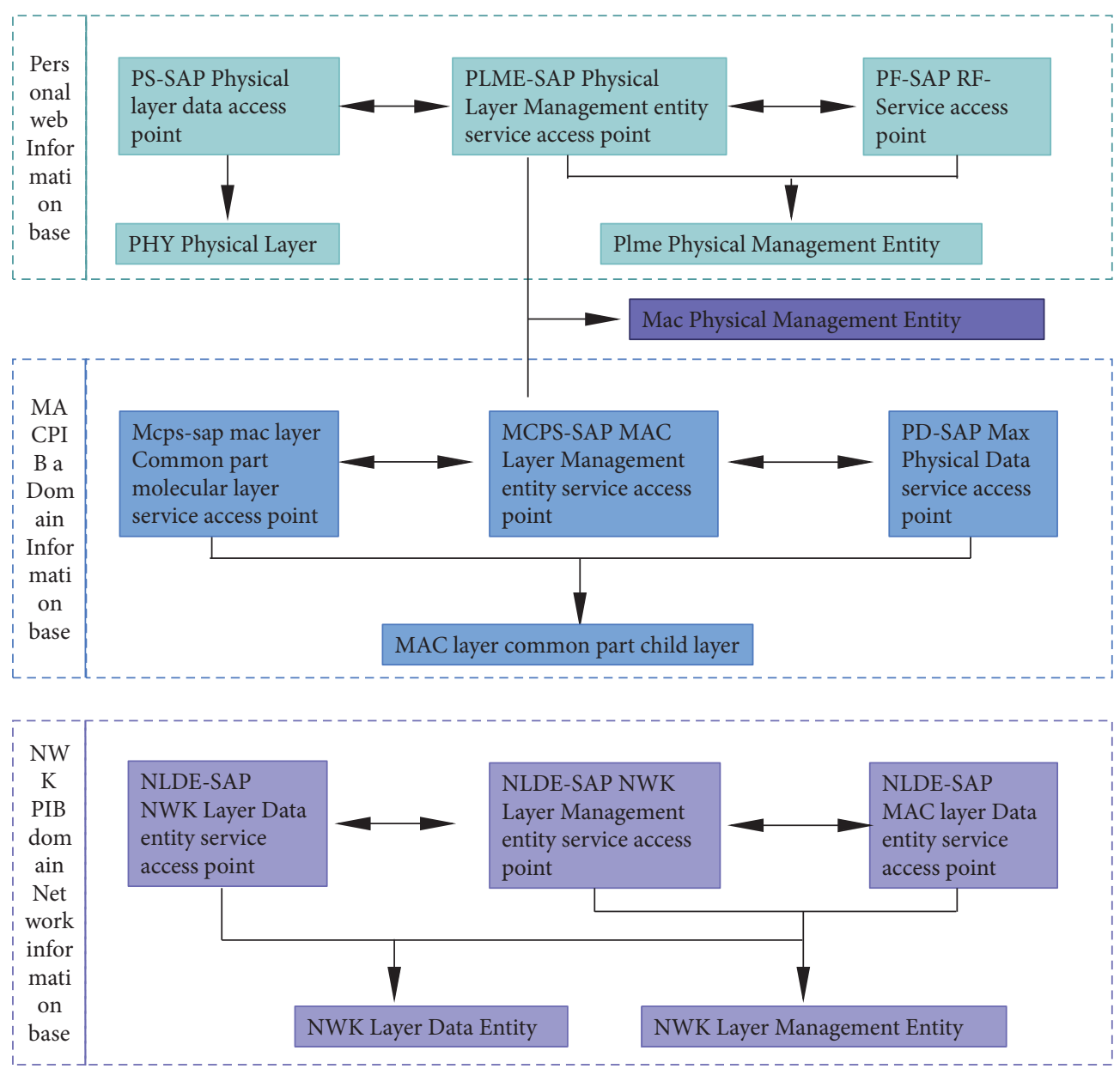

Figure 5: Schematic diagram of ZigBee protocol stack.

(3) Active RFID networks are less self-organizing.

(4) It is of high power consumption, small coverage, and long networking time.

(5) Based on the above analysis, it can be concluded that ZigBee can better perform the problem of workshop positioning.

4.2. ZigBee Protocol Stack. The ZigBee protocol standard adopts a hierarchical structure [17], and each layer provides a series of service functions. From the bottom to the top, they are physical layer (PHY), media access control layer (MAC), transport layer (TL), network layer (NWK), application layer (APL), and so on. The media access control layer and physical layer comply with the IEEE 802.15.4 standard. IEEE 802.15.4 is a standard for the IEEE wireless personal area network (PAN) working group [18].

In terms of protocol standardization, the bottom layer of the ZigBee protocol stack architecture is based on the IEEE 802.15.4 standard. The remaining protocols mainly refer to and apply existing standards. In other words, the underlying foundation of ZigBee is IEEE 802.15.4, and the IEEE handles only low-level MAC layer and physical layer protocols. On the basis of IEEE 802.15.4, the ZigBee Alliance is more eloquent in that it has developed a highly reliable, cost-effective network application specification and applied it to various electronic products to implement a low-speed wireless personal area network application [19]. In a sense, the media access control (MAC) layer and physical (PHY) layer of the ZigBee protocol stack have a unified standard: IEEE 802.15.4. ZigBee protocol stack diagram, shown in Figure 5.

\subsection{ZigBee Positioning Technology Analysis}

4.3.1. RISS Algorithm. Since the signal strength is attenuated in the propagation process, the distance between the transmitting node and the receiving node can be estimated based on the signal attenuation degree received by the receiving node [19]. The RSSI positioning technique [20] is a method for estimating the distance between nodes by measuring the degree of attenuation of the signal during propagation. The node signal attenuation model is represented by a logarithmic distance model,

$$
[\operatorname{Pr}(d)]_{d B m}=\left[\operatorname{Pr}\left(d_{0}\right)\right]_{d B m}-10 n \log \left(\frac{d}{d_{0}}\right)
$$

where $\mathrm{d}$ is the distance $(\mathrm{m})$ between two nodes (acceptor to transmitter); $\mathrm{d} 0$ is the unit reference distance $(\mathrm{m})$, and the 
value in this paper is $1 \mathrm{~m} ; \operatorname{Pr}(\mathrm{d})$ is the receiver end power of the received signal $(\mathrm{d} \mathrm{Bm}) ; \operatorname{Pr}(\mathrm{d} 0)$ is the received signal power $(\mathrm{d} \mathrm{Bm})$ received at the receiving end of the selected reference distance $\mathrm{d} 0$; and $\mathrm{n}$ is the path loss exponent [21]. For wireless sensors, the RSSI algorithm is affected by the environment. The distance between two different node pairs with the same RSSI value is different in the same network topology distribution, and the corresponding weights are also different. If the unknown node's position is calculated, only the RSSI value of the unknown node to a fixed known node is considered, and other correction methods are not added, which may result in a large error in the positioning result.

4.3.2. Weighted Trilateration Algorithm. At present, in the two-dimensional positioning, trilateration is a common positioning method. From the common knowledge of geometry, if the three reference nodes are not on the same straight line, then the distance between the unknown node and the reference node can uniquely determine the unknown node position. However, in the actual positioning process, the measurement error is unavoidable. Therefore, the three circles formed by the distance between the unknown node and the three reference nodes cannot be accurately intersected at one point. It is impossible to accurately find the unknown node. In general, the three-sided positioning effect is an area formed by using a reference node as the center of the circle and three circles measuring the radius as the radius. That is, the solution to the equation is that the range of the solution is within the scribe area, and the smaller the scribe area is, the smaller the solution is and the more accurate it is. Therefore, the relationship between the geometric position and the positioning accuracy of the reference node is studied in view of the low positioning accuracy of the general edgemeasuring algorithm. When the reference node is placed in the equilateral, the positioning error can achieve the minimum theoretical basis. The weighted information reflecting the geometric location of the reference node is introduced into the ordinary three-edge localization algorithm, and a weighted two-dimensional localization algorithm based on angle and side length information is improved to improve the localization accuracy.

(1) Weighting of Reference Nodes. In the triangle, let $\angle \alpha=$ $\left|\angle A-60^{\circ}\right|+\left|\angle B-60^{\circ}\right|$. If the triangle is an equilateral triangle, then $\angle \alpha$ is $0^{\circ}$, and the positioning effect is the best. If the triangle is an increasingly obtuse triangle, $\angle \alpha$ is also getting bigger and bigger, and A, B, and C become more and more. Approaching a straight line, the positioning accuracy is getting worse; when $\angle \alpha=240^{\circ}, \mathrm{A}, \mathrm{B}$, and C are three straight lines, and the unknown node cannot be located at this time. Therefore, this paper introduces the concept of weighting in the three-sided positioning. The angle weighting factor is $1 / \angle \alpha$. In addition, in addition to the interior angle of the triangle, the influence of the length of the side on the positioning should also be considered. If only the length of the AC is shortened, the value of $\angle \alpha$ does not change at this time. The impact of positioning is not enough. As BC becomes shorter and shorter, the difference in length between it and $\mathrm{AB}$ and $\mathrm{AC}$ gets larger and bigger. The three points $\mathrm{A}$,

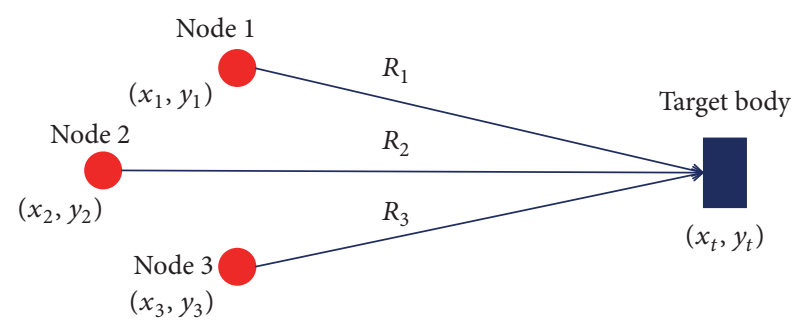

FIGURE 6: Diagram of solving equation coordinate.

$\mathrm{B}$, and $\mathrm{C}$ also get closer and closer to a straight line, so it is necessary to introduce weights on the sides factor.

(2) Calculation of Reference Nodes. In actual measurement, the RSSI value of the received signal decreases as the distance increases. When the distance is short, the RSSI value decreases faster, and as the distance increases, the RSSI value becomes more and more gradual and gradually cannot be reflected. The actual distance: therefore, in positioning, the unknown node should choose several reference nodes nearest to it to participate in positioning.

The trilateral positioning method [22] is one of the most widely used target positioning methods. As shown in the figure, the rectangle on the right side of the graph represents the target body, its position coordinates are unknown, and the three circles on the left side of the graph represent the reference nodes.

The position coordinates of these three points are known as $\left(x_{1}, y_{1}\right),\left(x_{2}, y_{2}\right)$, and $\left(x_{3}, y_{3}\right)$. Their distance to the target is $R_{1}, R_{2}$, and $R_{3}$. Set the position coordinates of the target $\left(x_{t}, y_{t}\right)$, with $R_{1}, R_{2}$, and $R_{3}$. It is known that an unknown quantity can be listed based on the distance formula between two points in the analytic geometry $\left(x_{t}, y_{t}\right)$ equation set

$$
\begin{aligned}
& \left(x_{1}-x_{t}\right)^{2}+\left(y_{1}-y_{t}\right)^{2}=R_{1}^{2} \\
& \left(x_{2}-x_{t}\right)^{2}+\left(y_{2}-y_{t}\right)^{2}=R_{2}^{2} \\
& \left(x_{3}-x_{t}\right)^{2}+\left(y_{3}-y_{t}\right)^{2}=R_{3}^{2}
\end{aligned}
$$

If this equation set is represented by a geometric figure, it is a circle with each reference node as its center and its distance from the reference node to the target body as its radius. The intersection point of the three circles is the position coordinate of the target body. Therefore, the position coordinates of the target body can be obtained by solving this equation group $\left(x_{t}, y_{t}\right)$. Now, the specific positioning process is shown in Figure 6.

Subtracting the third equation from the first two equations in the system of equations yields a new system of equations: $A I=b$.

$$
\begin{aligned}
A & =2\left(\begin{array}{ll}
x_{1}-x_{3} & y_{1}-y_{3} \\
x_{2}-x_{3} & y_{2}-y_{3}
\end{array}\right) \\
b & =\left(\begin{array}{l}
x_{1}^{2}-x_{3}^{2}+y_{1}^{2}-y_{3}^{2}+R_{3}^{2}-R_{1}^{2} \\
x_{2}^{2}-x_{3}^{2}+y_{2}^{2}-y_{3}^{2}+R_{3}^{2}-R_{2}^{2}
\end{array}\right)
\end{aligned}
$$




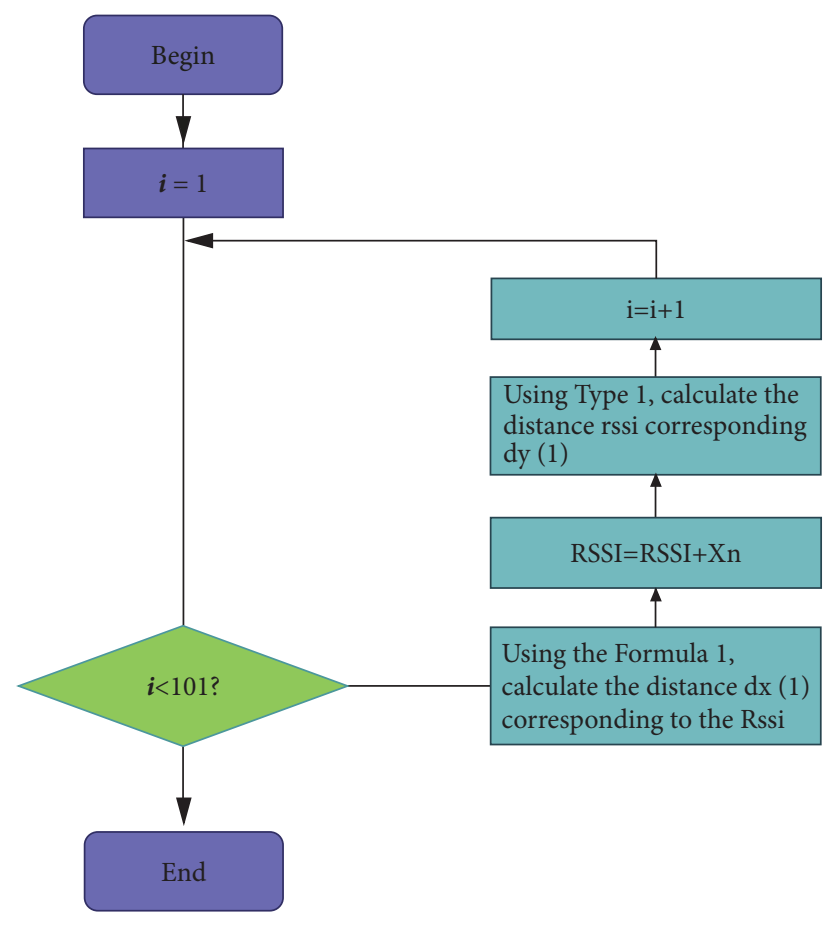

FIGURE 7: The derivation process of locating distance.

An approximate solution vector can be found using the principle of least squares.

$$
I=A^{T}\left(A^{T} * A\right)^{-1} * b
$$

Then I is used for the position vector of the target $\left(x_{t}, y_{t}\right)$.

4.4. Results Analysis. This paper simulates the positioning result and uses the distance value from the RSSI value to perform the positioning calculation. The simulation is divided into two parts.

The first part: the simulation verifies the difference between the distance value and the true distance obtained by RSSI with interference noise [23]. Simulation ideas: first calculate the corresponding RSSI value according to the distance; then, add Gaussian noise on the RSSI value to imitate the scene environment; finally, use the RSSI value with noise to push back the distance value compared with the original value of the distance [24]. Assume that the vector made from the original value is $\mathrm{dx}$ and the vector formed by the inversely derived distance value is $\mathrm{dy}$. $\mathrm{dx}$ vector value takes all integers from 1 to 100 ; $\mathrm{Xn}$ represents the noise in the field. Locate the distance flow as shown in Figure 7.

In the second part, the distance value obtained by further using the RSSI with interference noise is used as a positioning calculation to simulate the positioning effect of the ZigBee dedicated chip CC2431 in case of interference.

By extracting the coordinates $(x, y)$ of 100 points on a sinusoidal curve, the distance $\mathrm{d}$ from the reference node is calculated, and then the inverse distance value $\mathrm{d}^{\prime}$ is obtained by the above method; then the inverse distance value is used as the positioning basis, and the three-sided positioning algorithm finds that the coordinate values $\left(\mathrm{x}^{\prime}, \mathrm{y}^{\prime}\right)$ of some points are compared with their original coordinate values. This paper uses six sets of data to compare, as shown in Figure 8.

Simulation results: the six diagrams in Figure 8 are analyzed and compared. This paper lists the coordinates and actual coordinates obtained from positioning operations for six different workshops or different situations and consists of two sets of coordinates to form the two curves, respectively. Part of the positioning data curve detects the error between the positioning calculation coordinate value and the actual coordinate value. The results show that the overall effect of multiple sets of data is very close to the original value, the fluctuation range is not more than $2 \%$ of the whole, and the maximum error is not more than $1 \mathrm{~m}$, in line with the workshop positioning requirements.

\section{Conclusion}

The article constructs the workshop material networking. The realization of MES must be based on big data to build a network covering the whole production site, and the tracking location must adopt wireless network to realize the location data and production data needed to integrate and upload the big data by wired network, which can realize the communication function of MES. The most critical part of wireless location technology for MES system, the ZigBee positioning algorithm, is chosen, which is the RSSI of the signal intensity indication algorithm and the threeedge positioning algorithm, and the three-edge locating algorithm is applied to the CC2430 wireless MCU [25], and the location node is designed and implemented; then the 

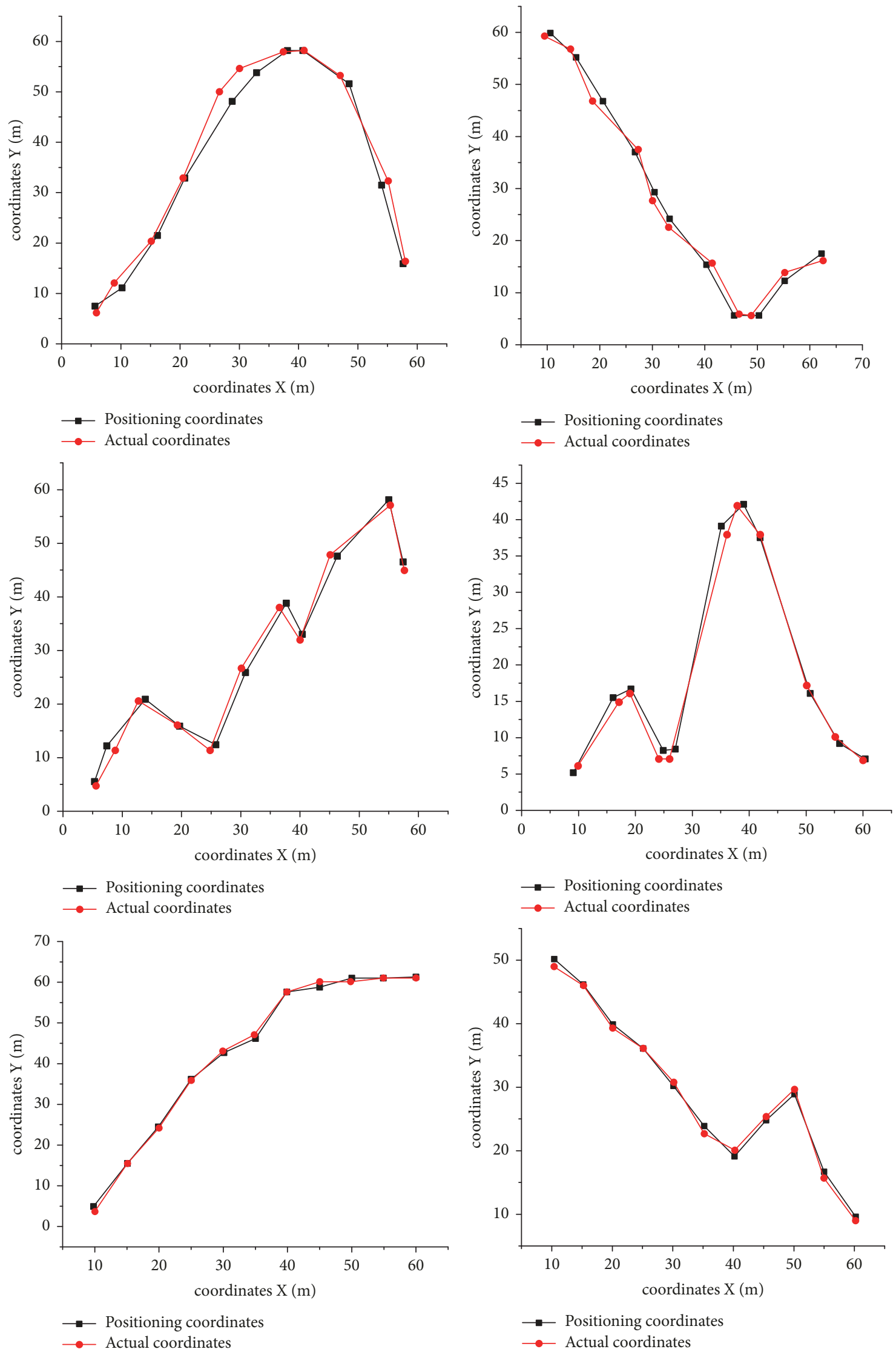

FIGURE 8: Location coordinates and actual coordinates. 
simulation experiment is carried out by the 6 sets of data. The maximum error between the locating coordinate and the actual coordinate is not more than 1 meter. Therefore, it can be concluded that this method can be used very well for the location of the workplace items.

\section{Data Availability}

The data used to support the findings of this study are available from the corresponding author upon request.

\section{Conflicts of Interest}

The authors declare that they have no conflicts of interest.

\section{Acknowledgments}

This work was supported by the Natural Science Foundation of Hebei (No. F2016209344).

\section{References}

[1] S. L. Chung and M. D. Jeng, "Manufacturing execution system (MES) for semiconductor manufacturing," in Proceedings of the IEEE International Conference on Systems, Man and Cybernetics, pp. 67-68, Yasmine Hammamet, Tunisia, 2002.

[2] J. Cai and C. Y. Fan, "Research on Intelligent Workshop Based on Industrial Internet of Things," China Educational Technology Equipment, vol. 9, no. 6, pp. 123-125, 2018.

[3] X. J. Liu, J. Y. Li, and L. N. Liu, "Internet-based textile workshop environment intelligent measurement and control system," Computer Applications, vol. 4, no. 6, pp. 24-28, 2015.

[4] Z. l. Fang, "PDA-Based Application and Research for Workshop Internet of Things," Green Computing and Communications IEEE, vol. 4, no. 6, pp. 99-101, 2013.

[5] S. Jiao, D. Song, Q. Zhang, and J. Tang, "Coal mine monitoring system based on ZigBee wireless sensor networks," Journal of Electronic Measurement and Instrument, vol. 27, no. 5, pp. 436442, 2013.

[6] C. Wei and M. J. Wang, "A comprehensive framework for selecting an ERP system," International Journal of Project Management, vol. 22, no. 2, pp. 161-169, 2004.

[7] B. F. Wu, Z. J. Chen, and C. T. Wang, "Research and Implementation of Data Acquisition Based on Manufacturing Execution System (MES)," China's Collective Economy, vol. 4, no. 6, pp. 8889, 2008.

[8] H. Sun, Y. B. Zhao, and M. H. Liang, "The Application of Internet of Things Technology in the Operating Room Management," China Digital Medicine, vol. 4, no. 6, pp. 97-101, 2016.

[9] Y. D. Fang, S. H. Ceng, and Y. Chen, "Technology Implementation of IOT in Workshop Manufacturing Execution System," Manufacturing Automation, vol. 4, no. 6, pp. 31-34, 2016.

[10] H. Chen, K. Fang, and Z. X. Wang, "ZigBee and other shortrange wireless communications technology and its application Comparison," Information Technology, vol. 4, no. 6, pp. 56-61, July 2015.

[11] B. Naz, "Evaluation and Analysis of Network Coding at Network Layer," in Proceedings of the International Conference on Progress in Informatics and Computing, vol. 4, pp. 34-36, July 2018.
[12] S. S. Zhou, X. G. Hu, and B. Wu, "Orbit determination and prediction accuracy analysis for a regional tracking network," Science China (Physics, Mechanics \& Astronomy), vol. 4, no. 6, pp. 21-25, 2010.

[13] K. Funabiki, T. Nishio, M. Morikura, K. Yamamoto, D. Murayama, and K. Nakahira, "ATRAS: adaptive MAC protocol for efficient and fair coexistence between radio over fiber-based and CSMA/CA-based WLANs," EURASIP Journal on Wireless Communications and Networking, vol. 19, no. 6, pp. 66-69, 2017.

[14] X. Lin, Z. G. Jia, S. B. Zhang, Z. L. Yang, and Y. Li, "ZigBee CC2430 MCU applications in a wireless temperature measurement system," Technological Innovation and Application, vol. 4, no. 6, pp. 24-28, 2017.

[15] Z. Wang, B. Zhang, and B. Wang, "Renewable energy consumption, economic growth and human development index in Pakistan: Evidence form simultaneous equation model," Journal of Cleaner Production, vol. 9, no. 6, pp. 132-134, 2018.

[16] G. W. Gao, G. Z. Bai, and Y. M. Yang, "Research on improved three edge localization algorithm based on RFID," Information Technology, vol. 4, no. 6, pp. 90-101, 2017.

[17] Y. Zhu and J. M. Lin, "Design and implementation of gateway based on Zig Bee wireless sensor networks," Information Technology, vol. 4, no. 6, pp. 34-38, 2007.

[18] S. S. Zhou, X. G. Hu, and B. Wu, "Orbit determination and prediction accuracy analysis for a regional tracking network," Science China (Physics, Mechanics \& Astronomy), vol. 53, no. 6, pp. 23-29, 2010.

[19] G. W. Gao and Y. M. Yan, "Research on improved three edge localization algorithm based on RFID," Information Technology, vol. 4, no. 6, pp. 17-22, 2017.

[20] X. Lin, J. Z. Guo, S. B. Zhang, Z. L. Yang, and Y. Li, “ZigBee CC2430 MCU applications in a wireless temperature measurement system," Technological Innovation and Application, vol. 3, no. 6, pp. 32-34, 2017.

[21] Z. Wang, B. Zhang, and B. Wang, "Renewable energy consumption, economic growth and human development index in Pakistan: Evidence form simultaneous equation model," Journal of Cleaner Production, vol. 184, pp. 1081-1090, 2018.

[22] G. Z. Gao, "Research on improved three edge localization algorithm based on RFID," Information Technology, vol. 4, no. 6, pp. 34-36, 2017.

[23] P. Balazki, K. Lindauer, J. Einloft, J. Ackermann, and I. Koch, "MONALISA for stochastic simulations of Petri net models of biochemical systems," BMC Bioinformatics, vol. 16, no. 1, 2015.

[24] J. Gu, Z. Gao, and W. Li, "Modeling of epidemic spreading with white Gaussian noise," Chinese Science Bulletin, vol. 56, no. 34, pp. 3683-3688, 2011.

[25] X. L. Zheng and J. Q. Fu, "Research on indoor location algorithm based on PDR and RSSI," Journal of Instrument and Instrument, vol. 21, no. 6, pp. 78-81, 2015. 


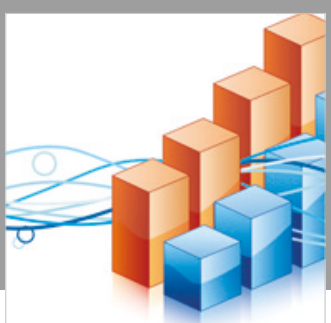

Advances in

Operations Research

\section{-n-m}
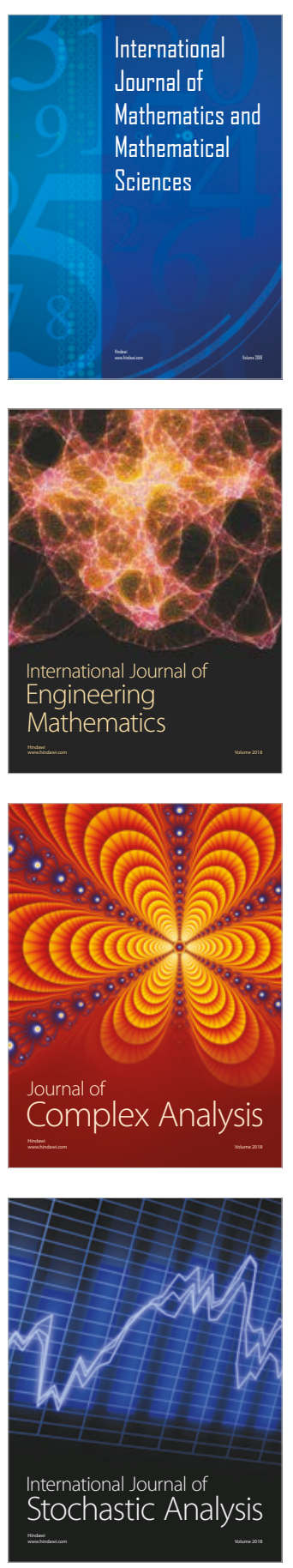
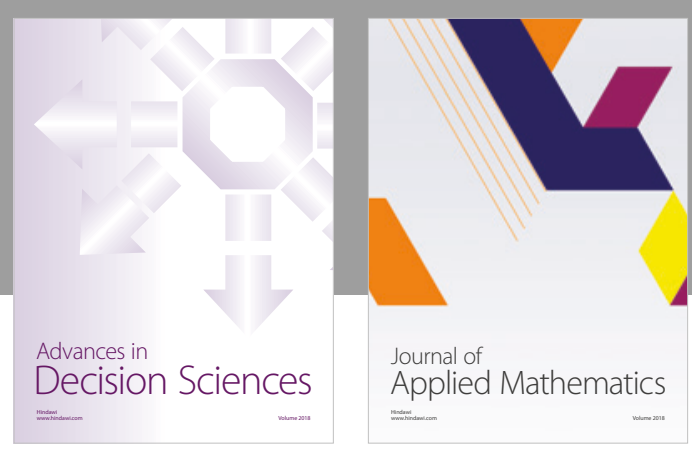

Journal of

Applied Mathematics
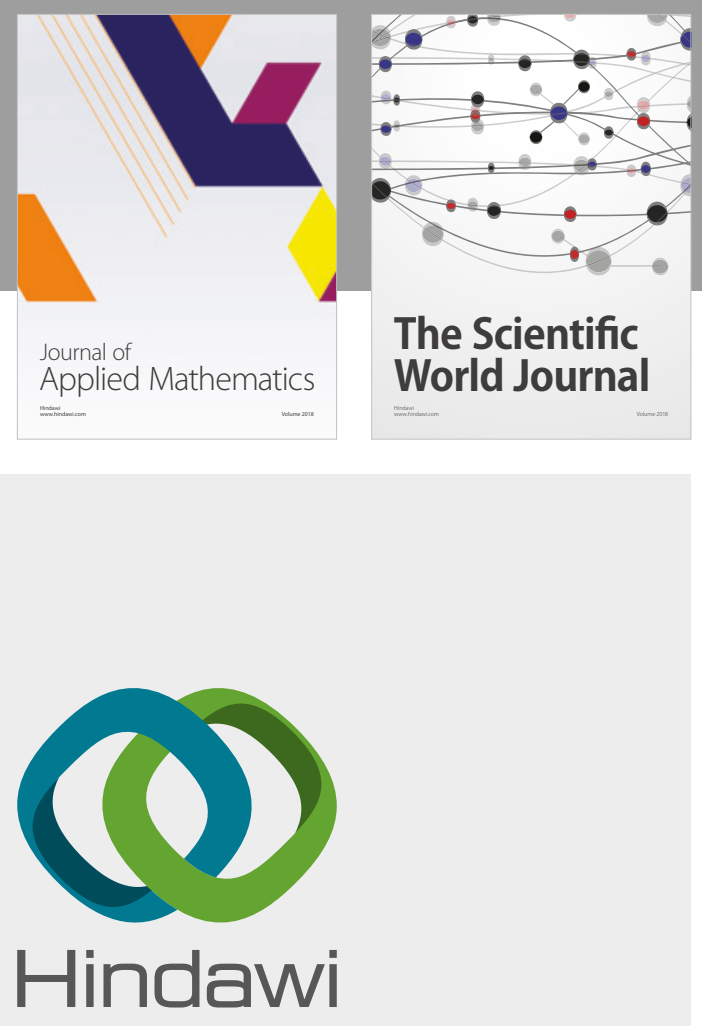

Submit your manuscripts at

www.hindawi.com

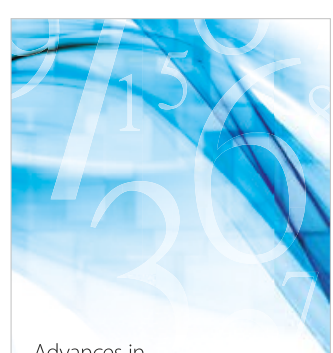

Advances in
Numerical Analysis
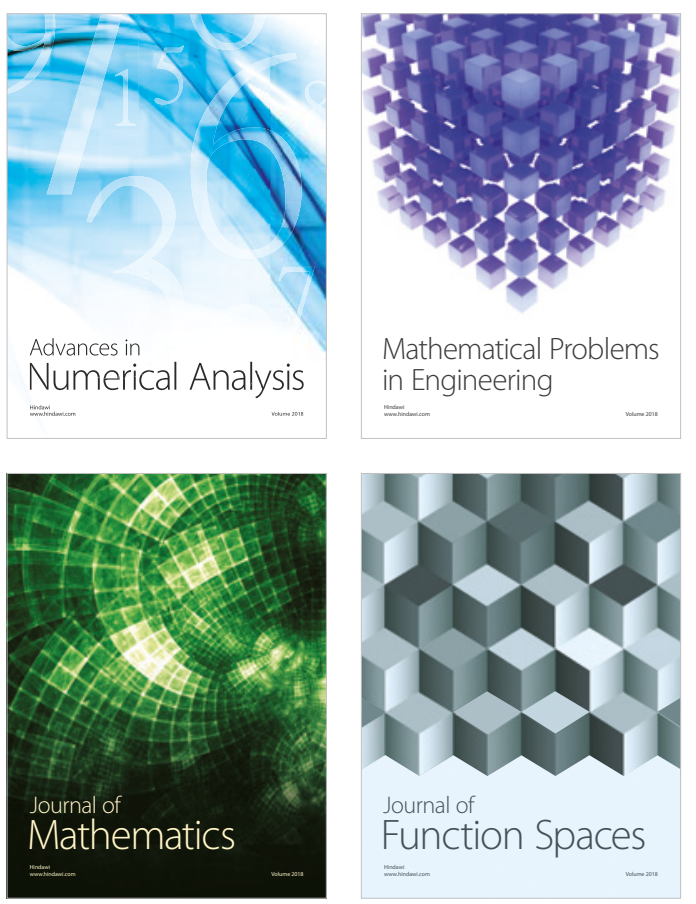

Mathematical Problems in Engineering

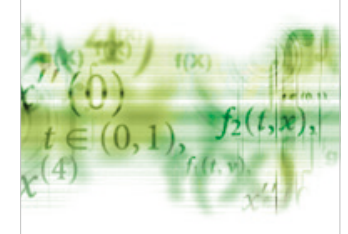

International Journal of

Differential Equations

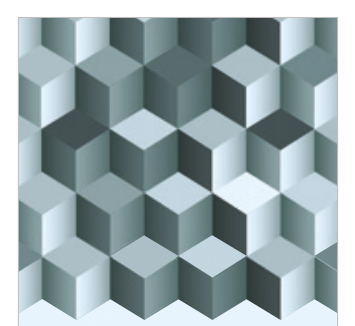

Journal of

Function Spaces
The Scientific

World Journal

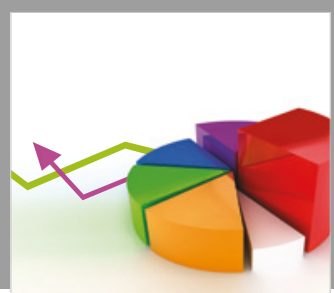

Journal of

Probability and Statistics
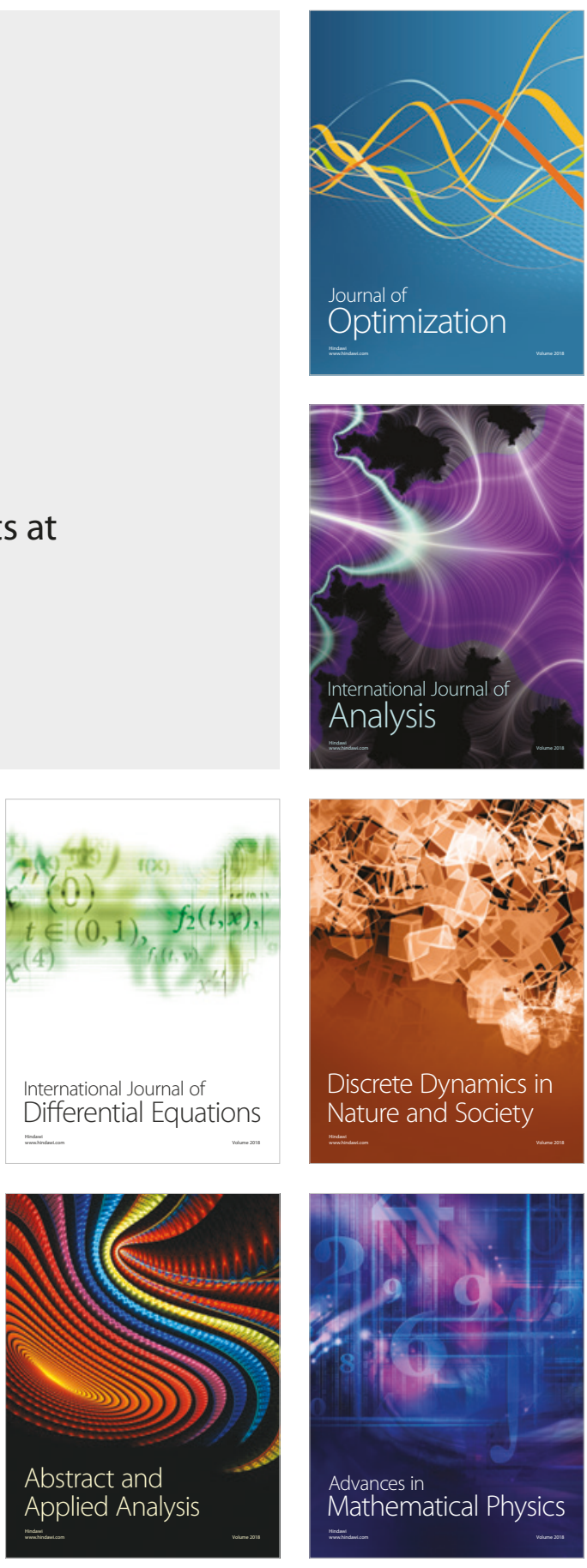